•综述・

\title{
激光雷达技术在动物生态学领域的研究进展
}

\author{
李 顺 邹亮宫一男杨海涛王天明冯利民 葛剑平*
}

(东北虎豹生物多样性国家野外科学观测研究站, 教育部生物多样性与生态工程重点实验室, 东北虎豹国家公园保护生态学

国家林草局重点实验室, 国家林草局东北虎豹监测与研究中心, 北京师范大学生命科学学院, 北京 100875)

摘要: 激光雷达(light detection and ranging, LiDAR)作为一门新兴的主动遥感技术, 近年来由于在提取和反演森林 参数水平上不断提高, 被越来越多地应用于动物生态学研究中。本文通过整理和搜集国内外文献, 对激光雷达的 技术特点及其在森林参数提取和动物生境上的研究进展进行综述, 指出当前基于LiDAR的森林参数反演算法主要 服务于森林资源调查或林学研究, 缺少对动物生态或生理意义相关的参数量化信息。目前该技术在国内的动物生 态学方面的应用较少, 尚未见文章发表。通过总结国外学者的研究, 分别从动物生境选择与三维森林结构的关系、 栖息地立体生境制图、生物多样性评估和物种分布模型预测三个方面综述了LiDAR在动物生态学研究中的应用现 状。相比传统方法, LiDAR技术提供的高精度三维结构信息, 能够显著提高动物生境质量的评估、生物多样性的监 测水平和物种分布模型的评价精度, 有利于从机理上加深对物种生境选择和集群过程的理解。但目前LiDAR技术 的应用主要集中在对已知的生态关系研究, 尤其是冠层结构与动物分布的关系, 缺少对林下层生活的动物生境质 量和生物多样性的监测和评估, 同时很多有关动物生存和繁衍与立体生境的关系研究有待从LiDAR数据中进一步 挖掘分析。未来应加强对森林林下层三维信息的提取, 提高林下层动物生境质量和生物多样性的监测水平, 同时 建立适用于动物生态和生理意义相关的参数, 为动物生境质量和生物多样性的评估提供标准的量化指标。

关键词：遥感; 动物生境监测; 物种分布; 生物多样性

\section{Advances in LiDAR technology in the field of animal ecology}

Shun Li, Liang Zou, Yinan Gong, Haitao Yang, Tianming Wang, Limin Feng, Jianping Ge*

Aumer Tiger and Leopard Biodiversity National Observation and Research Station, Ministry of Education Key Laboratory for Biodiversity Science and Ecological Engineering, National Forestry and Grassland Administration Key Laboratory for Conservation Ecology of Amur Tiger and Amur Leopard National Park, National Forestry and Grassland Administration Amur Tiger and Amur Leopard Monitoring and Research Center, College of Life Science, Beijing Normal University, Beijing 100875

Abstract: LiDAR (light detection and ranging), a fairly new active remote sensing technology, is being
widely used in the field of animal ecology by more and more scholars due to the recent development where
forest parameters can be extracted and inverted from LiDAR. In this paper, we review the advances in forest
parameter extraction from LiDAR and its many applications in studying wildlife habitat. We also analyze
current research on forest parameter inversion algorithms based on LiDAR, mainly in forestry research,
though we lack quantitative parameters related to the ecological significance of animals. Because few studies
have applied LiDAR technology to animal ecology research in China, we consider foreign research in this
field in three categories: (1) The relationship between species habitat selection and three-dimensional forest
structure; (2) Three-dimensional habitat mapping; (3) Biodiversity assessment and species distribution model
prediction. Compared with traditional methods, the high-precision three-dimensional structure information
provided by LiDAR can significantly improve the efficacy of monitoring animal habitat quality and
biodiversity and the modelling accuracy of species distribution models. These advancements contribute to
deeper understanding of species habitat selection and the clustering process mechanism. However, the studies
that utilize LiDAR to date have mainly focused on previously known ecological relationships, especially the

收稿日期: 2019-05-16; 接受日期: 2019-08-10

基金项目：国家自然科学基金(31842007)和科技部基础性工作专项基金(2016YF0500106)

* 通讯作者 Author for correspondence. E-mail: gejp@bnu.edu.cn 
relationship between canopy structure and species diversity. These studies fail to account for either forest understory habitat quality or biodiversity monitoring and evaluation. In short, the relationship between wildlife and its three-dimensional habitat needs to be further explored through analysis of LiDAR data. Future studies should focus on extracting three-dimensional structures of forest understories to improve the efficacy of monitoring habitat quality and biodiversity in the understory, and to provide standard quantitative indicators for the evaluation of animal ecology.

Key words: remote sensing; animal habitat monitoring; species distribution; biodiversity

野生动物是自然资源的重要组成部分, 同时在 自然生态系统中扮演着重要角色, 在种子传播和生 态系统功能调节等方面发挥着重要作用(Asner \& Levick, 2012)。然而, 人口的快速增长和自然资源的 不可持续利用, 导致野生动物生境大面积丧失和破 碎化, 野生动物种群数量和多样性急剧减少(Ripple et al, 2016)。因此, 如果想成功地保护地球上仅存的 具有重要生态学功能的物种, 就需要加强对珍稀物 种种群和栖息地的监测和评估, 获得更多关于物种 和栖息地的真实可靠数据。

遥感技术被广泛应用于野生动物种群监测和 栖息地生境质量评估, 主要包含两个方面: (1)野外 相机陷阱技术不断发展, 获得了大量野生动物的视 频和图片, 进一步丰富了关于野生动物分布、多度 和行为的资料(Burton et al, 2015; Xiao et al, 2016); (2)以卫星和飞机为平台获取的光学遥感影像数据, 侧重于研究土地利用和土地覆盖的变化带来的生 境退化和破碎化对动物种群和栖息地的影响 (McDermid et al, 2009; Bergl et al, 2012), 或是用于 监测动物的种群密度或迁徙、移动(Vermeulen et al, 2013)。近十年来, 以激光雷达(light detection and ranging, LiDAR)为代表的主动遥感技术由于其提供 的高精度三维地物信息而在动物生态学中得到了 广泛应用, 尤其在获取动物三维结构生境和动物多 样性研究方面具有优势(Bergen et al, 2009; Davies \& Asner, 2014; Simonson et al, 2014)。

LiDAR技术因其不依赖于太阳辐射可以昼夜 工作，同时可以根据探测目标的不同选择不同的电 磁波波长和发射方式等优点，已经在林业、农业、 气象、测绘和考古等领域得到广泛应用(郭庆华等, 2018)。20世纪90年代后期, 随着全球定位系统 (Global Positioning System, GPS)及惯性导航系统 (Inertial Navigation System, INS)的发展, 激光雷达 技术能够快速大面积地获取森林结构信息, 如树木
定位、树高估计、树冠体积估算、森林地上生物量 反演等，使激光雷达技术成为森林资源调查的重要 手段, 为森林生态学研究和森林经营管理提供了大 量的参数信息(李增元等, 2016)。

森林生态系统的三维结构是影响动物群落组 成的一个重要因素, 林栖野生动物都生活在一个立 体的生境结构中(Flaspohler et al, 2010; Palminteri et al, 2012; Müller et al, 2014)。植被的林冠层是众多灵 长类和鸟类动物的主要栖息场所(McLean et al, 2016; Davies et al, 2017), 而林下层也是许多兽类的 主要活动空间(Serrouya \& D’Eon, 2008; Psyllakis \& Gillingham, 2009; Burkepile et al, 2013; Blakey et al, 2017), 这说明森林结构在动物生境选择中具有重 要的作用。

然而森林生态系统结构的多样性、复杂性以及 差异性的描述和预测是十分复杂的, 传统的遥感技 术或人工调查手段仅限于描述森林结构的平面信 息(如郁闭度、盖度、林分密度等)及其变化过程, 对 于复杂的三维结构特征(冠层垂直结构、树高、林下 垂直结构等), 很难进行直观的描述并建立统一的 量化标准。而LiDAR技术能够形象并且高效地获取 森林结构的三维特征, 与传统的遥感技术相比, 在 监测和评估物种栖息地方面具有不可替代的优势, 有助于获得更加直观的物种栖息地环境数据, 进而 更深入地探讨动植物之间的关系, 丰富动物生态学 和群落生态学相关知识的研究。已有研究指出, 未 来动物生态学的研究将因考虑物种的三维生境结 构而获得更深的认识(Davies \& Asner, 2014)。

但是目前激光雷达在动物生态学领域的应用 研究相对零散, 主要受技术驱动。本文通过阐述激 光雷达的技术特点, 对其在动物生态学(本文综述 的动物类群只包含陆地动物)中的应用现状进行综 述，探讨该技术在我国动物生态学领域的应用前景 和发展方向, 为该技术在我国相关领域中的应用研 
究提供一些参考。

\section{激光雷达的特点及应用}

\section{1 激光雷达技术的原理和优势}

激光雷达是激光探测与测距系统的简称, 它通 过传感器发出的信号在传感器与目标物之间的传 播距离, 分析目标地物表面的反射能量、波谱和相 位信息, 进行目标定位的精确解算, 从而呈现目标 物精确的三维结构信息(郭庆华等, 2018; 王瑶瑶等, 2018)。

激光雷达可通过记录完整的回波波形(大光斑: 10-100 m)反演出森林的垂直结构与生物量(马利群 等，2011); 或是记录少量的离散回波(小光斑: 0.1-1.0 m), 利用高密度的激光点云数据, 进行精确 的单木高度估测(庞勇等, 2008)。激光雷达在一些关 键技术指标上远远超越了传统的人工调查方法, 其 优势在于: (1)精确度高, 激光雷达通过发射完整的 信号和记录回波数据, 可以获得精确标准的量化信 息, 很大程度上减小了人工估测带来的误差; (2)植 被穿透能力强, 激光在植被中传播时, 可以在树 冠、树枝、地面等多个高程发生反射，从而得到多 次回波数, 这是其他雷达所不具备的优势, 特别是 得到的地面回波数据, 有效克服了植被影响, 使精 确探测地面真实地貌特征成为可能(张健等, 2011); (3)激光雷达获取的点云数据记录了探测地物的三 维坐标信息, 不仅能还原真实的三维场景信息, 还 能进行数字化, 为后续森林结构指标的量化和建模 提供数据支持。

\section{2 激光雷达搭载平台及数据处理软件}

依据搭载平台的不同, 激光雷达可以分为星载 激光雷达(spaceborne lidar)、机载激光雷达(airborne laser scanner, ALS)、无人机激光雷达(drone laser scanner, DLS)、车载激光雷达(vehicle-mounted laser scanner, VLS) 和地基激光雷达 (terrestrial laser scanner, TLS)。从星载激光雷达到地基激光雷达, 扫 描获取的效率越来越低, 但精度越来越高, 可以满 足从全球区域尺度到立地尺度上的研究需求(郭庆 华等, 2018)。地基激光雷达也称为地面激光雷达扫 描系统, 通常用于单一目标或小尺度上精细三维数 据的采集。机载或无人机激光雷达以飞行器为搭载 平台，通常用于区域尺度三维信息数据的快速获取，
其核心部件包括激光雷达传感器、全球定位系统和 惯性测量单元。在林业调查中, 应用较广主要是地 基激光雷达、无人机激光雷达和机载激光雷达，可 以获取从单木到林分水平上的高精度三维信息, 为 提供真实的三维森林场景和实现林分几何结构参 数的自动获取提供了可能。

从激光雷达扫描仪获取的原始数据为目标地 物的点云数据(point cloud data), 记录了包括每个空 间点的三维坐标、反射强度、回波次数和扫描时的 角度信息。现在有很多国内外开发的专业软件能够 更高效、智能地处理激光雷达获取的点云数据, 包 括免费的开源软件和商业软件(表1)。

\section{2 基于激光雷达的森林参数提取及其在动物} 上的应用

为了及时准确掌握森林资源信息，众多学者已 在基于LiDAR的森林结构和生态系统功能参数的 算法提取和反演上有深入的研究, 尤其是基于机载 激光雷达、无人机激光雷达和地基激光雷达平台的 单木分割算法为基础的众多森林参数信息(表2)。单 木分割即从激光点云数据中提取和识别每棵树木 (图1), 它是获取单木尺度森林结构参数的重要基 础。近几年来, 单木分割算法成为激光雷达林业研 究的热点, 主要包括两大类算法: (1)以冠层高度模 型(Canopy Height Model, CHM)为基础, 对CHM模 型进行区域分割; (2)根据树木几何结构直接基于点 云分割的算法。主要分割方法的比较见表2。

除了单木分割算法, 许多学者还提取和反演了 一系列森林群落结构和生态系统功能的参数, 如树 冠高度(Thomas et al, 2006; Coops et al, 2007)、叶面 积指数和郁闭度 (Kwak \& Cho, 2012; Zhao et al, 2015)、冠层垂直结构(Jaskierniak et al, 2011)、地上 生物量(Clark et al, 2011)等。其中很多森林参数对动 物生境质量的量化和评估具有重要意义(表3)。

但是目前基于 LiDAR的森林参数算法主要是 服务于森林资源的调查或森林生态学的研究, 与动 物生境并没有直接的关系，所对应的动物生态或生 理意义较为模糊。且主要提取和研究的对象为森林 中的冠层或乔木层, 缺少对林下层的植被及微地形 结构的参数提取和反演, 缺乏对生活在林下层的野 生动物生境的量化和评估能力。 
表1 国内外主流激光雷达数据处理软件

Table 1 The main softwares for LiDAR data processing of both domestic and overseas

\begin{tabular}{|c|c|c|}
\hline 软件 Software & 类型 Type & 主要应用及特点 Application and characteristics \\
\hline TerraSolid & $\begin{array}{l}\text { 商业软件 } \\
\text { Commercial software }\end{array}$ & $\begin{array}{l}\text { 目前国内外航测部门广泛采用的软件, 用于点云、影像处理。A widely used software in aerial } \\
\text { survey department at present, used for point cloud and image data processing. }\end{array}$ \\
\hline NVI LiDAR & $\begin{array}{l}\text { 商业软件 } \\
\text { Commercial software }\end{array}$ & $\begin{array}{l}\text { 可以自动提取DEM/DSM/建筑物/植被等三维模型, 提取的信息可在其他平台(如ENVI、ArcGIS、 } \\
\text { Google Earth)进一步使用和分析, 提供二次开发接口。Automatically extracted 3D models such as } \\
\text { DEM/DSM/buildings/vegetation, the extracted data can be further used and analyzed on other } \\
\text { platforms (such as ENVI, ArcGIS and Google Earth), and provide secondary development interface. }\end{array}$ \\
\hline $\begin{array}{l}\text { Global Mapper } \\
\text { LiDAR Module }\end{array}$ & $\begin{array}{l}\text { 商业软件 } \\
\text { Commercial software }\end{array}$ & $\begin{array}{l}\text { 集成了一系列全面的点云处理工具和数据访问接口, 包括点云生成、自动分类及要素提取。 } \\
\text { Integrated a series of comprehensive point cloud processing tools and data access interface, including } \\
\text { point cloud generation, automatic classification and element extraction. }\end{array}$ \\
\hline astools & $\begin{array}{l}\text { 商业软件 } \\
\text { Commercial software }\end{array}$ & $\begin{array}{l}\text { 提供高效点云处理算法的库, 支持点云格式转换、点云处理等常见的算法功能。Providing } \\
\text { efficient point cloud processing algorithm library, and supporting the functions of point cloud format } \\
\text { conversion, point cloud processing and other common algorithm. }\end{array}$ \\
\hline loud Compare & $\begin{array}{l}\text { 开源软件 } \\
\text { Open source software }\end{array}$ & $\begin{array}{l}\text { 提供了一些基本工具用于手动编辑和呈现3D点云数据及各种处理算法, 用于实现点云距离计 } \\
\text { 算、空间统计分析、点云分割和几何特征估算。Providing some basic tools for manually editing and } \\
\text { rendering 3D point cloud data and various processing algorithms, which are used to realize point cloud } \\
\text { distance calculation, spatial statistical analysis, point cloud segmentation and geometric feature } \\
\text { estimation. }\end{array}$ \\
\hline Fusion & $\begin{array}{l}\text { 开源软件 } \\
\text { Open source software }\end{array}$ & $\begin{array}{l}\text { 适合于林学和生态学研究, 可以提取多种点云获取的林业参数信息。Suitable for the study of } \\
\text { forestry and ecology, and can extract the information of forestry parameters obtained from various } \\
\text { point clouds. }\end{array}$ \\
\hline RiALITY & $\begin{array}{l}\text { 商业软件 } \\
\text { Commercial software }\end{array}$ & $\begin{array}{l}\text { 可在iPad上运行激光雷达数据可视化功能, 支持真彩色三维点云和导航功能。It can run LiDAR } \\
\text { data visualization on the iPad, supporting true color 3D point cloud and navigation. }\end{array}$ \\
\hline LP360 & $\begin{array}{l}\text { 商业软件 } \\
\text { Commercial software }\end{array}$ & $\begin{array}{l}\text { 桌面软件, 可以独立或在ArcGIS环境中实现, 提供不同解决方案, 从快速可视化到一些扩展线 } \\
\text { 产品, 包括地面点云自动分类和信息提取。Desktop software, which can be implemented } \\
\text { independently or in ArcGIS environment, providing different solutions, from rapid visualization to } \\
\text { some extension line products, including ground point cloud automatic classification and information } \\
\text { extraction. }\end{array}$ \\
\hline FME & $\begin{array}{l}\text { 商业软件 } \\
\text { Commercial software }\end{array}$ & $\begin{array}{l}\text { 由加拿大Safe Software公司研发, 最大特点是支持超过 } 300 \text { 多种空间数据格式及相互转换, 包括 } \\
\text { 点云数据。Developed by Company of Safe Software, Canada, supporting for more than } 300 \text { spatial } \\
\text { data formats and conversions, including point cloud data. }\end{array}$ \\
\hline LiDAR 360 & $\begin{array}{l}\text { 商业软件 } \\
\text { Commercial software }\end{array}$ & $\begin{array}{l}\text { 由国内数字绿土公司研发, 有针对城市规划、林业、电力等不同行业应用需求的信息提取和模块 } \\
\text { 分析, 尤其是针对林业应用的单木分割算法, 能够进行点云数据信息挖掘和超大数据处理功能。 } \\
\text { Developed by Green Valley Company in China, focusing on information extraction and analysis module } \\
\text { for urban planning, forestry and electric power application, especially for forestry application of tree } \\
\text { segmentation algorithm, with point cloud data mining and data processing functions. }\end{array}$ \\
\hline
\end{tabular}

表2 主要单木分割算法比较

Table 2 Comparison of the main algorithms of single tree segmentation

\begin{tabular}{|c|c|c|c|}
\hline $\begin{array}{l}\text { 方法 } \\
\text { Methods }\end{array}$ & $\begin{array}{l}\text { 参考文献 } \\
\text { Reference }\end{array}$ & $\begin{array}{l}\text { 树种 } \\
\text { Tree species }\end{array}$ & $\begin{array}{l}\text { 分割精度 } \\
\text { Accuracy of segmentation }\end{array}$ \\
\hline 区域增长 Region growing & Hyyppa et al, 2001 & 针叶林 Coniferous forest & - \\
\hline “注水”算法 Pouring algorithm & Koch et al, 2006 & $\begin{array}{l}\text { 针叶林、阔叶林 } \\
\text { Coniferous forest and broad-leaved forest }\end{array}$ & $62 \%$ \\
\hline 标记分水岭 Marked watershed & Chen et al, 2006 & 阔叶林 Broad-leaved forest & $64 \%$ \\
\hline K均值聚类 K-means clustering & Morsdorf et al, 2004 & 针叶林 Coniferous forest & $61 \%$ \\
\hline $\begin{array}{l}\text { 图论归一化分割 } \\
\text { Graph theory normalized segmentation }\end{array}$ & Reitberger et al, 2009 & $\begin{array}{l}\text { 针叶林、阔叶林 } \\
\text { Coniferous forests and broad-leaved forest }\end{array}$ & $66 \%$ \\
\hline $\begin{array}{l}\text { 基于区域增长和阈值判断结合 } \\
\text { Based on the combination of regional } \\
\text { growth and threshold judgment }\end{array}$ & Li et al, 2012 & 针阔混交林 Theropencedrymion & $94 \%$ \\
\hline 相对最短路径 Relative shortest path & Tao et al, 2015 & $\begin{array}{l}\text { 针叶林、阔叶林 } \\
\text { Coniferous forest and broad-leaved forest }\end{array}$ & $83 \%-93 \%$ \\
\hline
\end{tabular}





图1 基于相对最短路径算法的样地尺度单木分割效果。(a)俯视图; (b)正视图。获取平台: 地基激光雷达; 获取时间: 2017 年; 获取地点: 东北虎豹国家公园东部地区。

Fig. 1 Results of individual tree segmentation in plot scale by the relative shortest path algorithm based on terrestrial laser scanner. (a) Top view; (b) Front view. Platform: TLS; Captured time: 2017; Location: Eastern of National Park for Amur Tiger and Leopard

\section{表3 激光雷达提取的用于动物生境研究的主要森林参数}

Table 3 Forest parameters extracted by LiDAR for wildlife habitat application studies

\begin{tabular}{|c|c|c|c|}
\hline $\begin{array}{l}\text { 森林参数 } \\
\text { Forest parameters }\end{array}$ & $\begin{array}{l}\text { 参数描述 } \\
\text { Parametric description }\end{array}$ & $\begin{array}{l}\text { 与动物生境关系 } \\
\text { Relationship with wildlife habitat }\end{array}$ & $\begin{array}{l}\text { 参考文献 } \\
\text { Reference }\end{array}$ \\
\hline $\begin{array}{l}\text { 郁闭度 } \\
\text { Canopy cover }\end{array}$ & $\begin{array}{l}\text { 指森林中乔木树冠在阳光直射下在地面的 } \\
\text { 总投影面积(冠幅)与此林地(林分)总面积的 } \\
\text { 比, 反映林分的密度。The ratio of the total } \\
\text { projected area (canopy width) of the canopy } \\
\text { on the ground under direct sunlight to the total } \\
\text { area of the forest (forest stand), which reflects } \\
\text { the density of the forest stand. }\end{array}$ & $\begin{array}{l}\text { 大部分研究表明, 鸟类与蝙蝠类的活动与郁闭度高 } \\
\text { 度相关; 有蹄类动物的季节选择与郁闭度相关。Most } \\
\text { studies show that the activities of birds and bats are } \\
\text { highly correlated with canopy density. Seasonal sele- } \\
\text { ction of ungulates is related to canopy cover. }\end{array}$ & $\begin{array}{l}\text { Garabedian et al, } \\
\text { 2014; Lone et al, } \\
\text { 2014; Ewald et al, } \\
\text { 2014; Melin et al, } \\
\text { 2016b; Blakey et } \\
\text { al, } 2017\end{array}$ \\
\hline $\begin{array}{l}\text { 冠层高度 } \\
\text { Canopy height }\end{array}$ & $\begin{array}{l}\text { 是森林垂直生境结构的重要参数, 反映的是 } \\
\text { 森林冠层距离地面的平均高度。An } \\
\text { important parameter of forest vertical habitat } \\
\text { structure, which reflects the average height of } \\
\text { forest canopy from the ground. }\end{array}$ & $\begin{array}{l}\text { 已有一些具有气候依赖性的鸟类在不同季节和不同 } \\
\text { 气候条件下栖息的冠层高度不同; 鸟类和蝙蝠类动 } \\
\text { 物的活动和占域在不同冠层高度上也呈现异质性。 } \\
\text { Some climate-dependent birds have different canopy } \\
\text { heights in different seasons and climate. The activities } \\
\text { and habitats of birds and bats are also present heter- } \\
\text { ogeneous at different canopy heights. }\end{array}$ & $\begin{array}{l}\text { Bradbury et al, } \\
\text { 2005; Goetz et al, } \\
\text { 2010; Garabedian } \\
\text { et al, 2017; Blakey } \\
\text { et al, } 2017\end{array}$ \\
\hline $\begin{array}{l}\text { 冠层垂直分布 } \\
\text { Canopy vertical } \\
\text { distribution }\end{array}$ & $\begin{array}{l}\text { 冠层部分在不同高度层枝叶的结构和密度 } \\
\text { 分布情况。The structure and density distri- } \\
\text { bution of canopy in different height layers. }\end{array}$ & $\begin{array}{l}\text { 目前研究表明, 灵长类动物个体生境利用与冠层垂 } \\
\text { 直分布相关。Current studies have shown that habitat } \\
\text { use of individual primate is related to vertical canopy } \\
\text { distribution. }\end{array}$ & $\begin{array}{l}\text { Palminteri et al, } \\
\text { 2012; Davies et al, } \\
2019\end{array}$ \\
\hline $\begin{array}{l}\text { 林下层密度 } \\
\text { Understory } \\
\text { density }\end{array}$ & $\begin{array}{l}\text { 单位面积内树冠以下部分枝叶和灌草的分 } \\
\text { 布密集程度。Distribution density of branches, } \\
\text { leaves and shrubs in the understory within the } \\
\text { unit area. }\end{array}$ & $\begin{array}{l}\text { 林下层密度关系到林下哺乳动物食物资源丰富度, } \\
\text { 同时影响动物休息、捕猎和产仔等其他行为选择。The } \\
\text { density of understory is related to the abundance of food } \\
\text { resources of understory mammals, and also affects other } \\
\text { behavioral choices such as resting, hunting and } \\
\text { breeding. }\end{array}$ & $\begin{array}{l}\text { Loarie et al, 2013; } \\
\text { Davies et al, 2016; } \\
\text { Melin et al, 2016a }\end{array}$ \\
\hline $\begin{array}{l}\text { 水平结构 } \\
\text { Horizontal } \\
\text { structure }\end{array}$ & $\begin{array}{l}\text { 植被在二维平面上的结构状况, 包括郁闭度 } \\
\text { 和灌草层覆盖度等。The structure of veg- } \\
\text { etation on the two-dimensional plane includes } \\
\text { canopy cover and cover of shrub and herb. }\end{array}$ & $\begin{array}{l}\text { 多数研究表明, 植被水平结构的多样性与动物物种 } \\
\text { 多样性具有正相关关系。Most studies show that the } \\
\text { diversity of horizontal structure of vegetation is posi- } \\
\text { tively correlated with the diversity of animal species. }\end{array}$ & $\begin{array}{l}\text { Flaspohler et al, } \\
2010\end{array}$ \\
\hline
\end{tabular}




\section{LiDAR在物种生境监测及生物多样性中自}

\section{1 物种生境选择与三维森林结构的关系}

了解动物的生境选择是对野生物种进行管理 和保护的重要前提。很多研究认为植被结构是影响 动物生境选择的重要因素(Carlson, 2000; Takahata et al, 2014)。主要表现在三个方面: (1)森林结构直接 决定了食物资源的分布, 食物资源在时空尺度上分 布的差异性对脊椎动物的分布有着显著的影响, 尤 其是对森林中依赖果实和树叶为主要食物的动物 (Garber, 1987; Saracco \& Groom, 2004); (2)森林的空 间结构塑造了栖息地不同的通透程度，显著地影响 到林栖动物的食物获取(Ren et al, 2001)、躲避捕食 者的能力(Lone et al, 2014)、空间通过能力或动物的 运动模式(Palminteri et al, 2012; McLean et al, 2016; Davies et al, 2017)以及睡眠或休息地点的选择(Cui et al, 2006; Xiang et al, 2010; Chu et al, 2018)等诸多 方面; (3)森林的三维结构通过改变微气候条件(气 温、湿度、光照、风速等), 影响栖息地的物理环境 变化, 进而影响物种的生境选择(Chen et al, 1999; Ameztegui \& Coll, 2015)。但是目前关于栖息地的三 维森林结构特征信息十分贵乏, 尤其是群落及区域 尺度上的植被结构与动物的生理、行为、种群分布 和种间关系的影响过程尚不十分明确。LiDAR技术 可提供独有的精细三维结构重建功能, 通过对生境 结构的具体量化, 有助于从机理上探讨动植物之间 的影响过程。

植被的林冠层是很多动物的主要栖息场所, 林 冠层的结构与动物的生境选择密切相关。Davies等 (2017)通过机载激光雷达获取的高分辨率冠层结构 数据揭示了不同性别和年龄的婆罗洲猩猩(Pongo pygmaeus) 个体在生境破碎化的马来西亚热带森林 地区的运动模式, 发现它们虽然利用了受干扰的森 林, 但它们往往选择具有某些特定属性的冠层结构, 这表明并非所有受干扰或退化的森林都对猩猩种 群的长期可持续发展具有同等价值。Garabedian等 (2017)利用LiDAR获取的精细分辨率数据评估了北 美濒危鸟类红头豚木鸟(Leuconotopicus borealis)资 源选择的生境属性(胸径、基径面积和郁闭度), 确定 了该物种受食生境恢复的最低阈值, 通过确定与阈 值响应相关的全部森林结构条件来指导森林鸟类 的保护。Bradbury等(2005)通过LiDAR技术模拟鸟
类与植被结构的关系发现, 在英国灌木林中, 大山 雀(Parus major)卵孚化卵成功率在反常的暖春随冠层 高度增加, 而在冷春则相反。该研究通过激光雷达 技术在精确探测植被结构上的优势，揭示了鸟类生 存质量与气候之间的关系。

除了冠层，林下层也是许多动物的主要活动空 间，其植被结构直接关系到兽类的生境选择和活 动。例如, Melin等(2016b)通过机载激光雷达描述了 芬兰西部地区雌性驼鹿(Alces alces)一年内生境选 择变化的精细三维特征，获得了不同性别和年龄的 驼鹿在生境利用模式上的差异性, 尤其是关于森林 结构对产仔雌鹿的影响, 相对于传统森林调查方法 获得了更新和更准确的信息。Blackey等(2017)采用 地基激光雷达调查林冠以下蝙蝠 (Vespertilio superans)群落分布与森林结构之间的关系，研究表 明树干的密度(stem density)是反映蝙蝠与环境关系 最强的预测因子，同时该研究首次将LiDAR衍生的 三维森林结构指标与不同种类蝙蝠的特征联系起 来, 结果显示不同蝙蝠的活动特征具有明显差异性, 相对于叫声、体型或翅膀, 森林结构和受食策略更 能解释这种差异性的来源。Loarie等(2013)采用机载 LiDAR技术在南非克鲁格国家公园对狮子(Panthera leo)的三维环境特征进行描述，通过量化的方法 验证了植被结构决定捕食与被捕食关系的假设, 结 果显示由于雌狮采取围攻的捕猎方式倾向于选择 视野开阔的森林结构, 而雄狮采取伏击的捕猎方式 一般选择视野较窄的植被茂密区。

这些研究表明, 激光雷达技术在量化动物生境 选择的具体环境特征时具有很大的优势，对于进一 步挖掘动物生境选择与植被结构关系的机理有很 大的作用。从当前的研究趋势看, 对于动物生境选 择的研究, 逐渐从动物生境利用与森林结构之间关 系的统计观察转向机理特性研究 (McGill et al, 2006), 这也是动物和群落生态学研究的关键一步, 未来激光雷达技术将在刻画动物与森林结构的机 理关系方面发挥很大的潜力。

\section{2 栖息地立体生境制图}

传统的动物生境制图更多地依靠中分辨率的 光学遥感数据, 获得一些平面化的参数信息(植被 指数、冠层郁闭度、土地利用类型等)来量化生境特 征，而一些特殊的生境变量如冠层垂直结构、林下 垂直结构、地形起伏变化等很难通过光学遥感精确 
测定，依靠卫星和飞机平台的激光雷达系统能够提 供大尺度的三维冠层垂直结构和地形信息, 近年来 国外应用激光雷达数据进行野生动物三维生境制 图和野生动物管理上逐渐增加(Hyde et al, 2005, 2006; Martinuzzi et al, 2009; Olsoy et al, 2018)。如果 将激光雷达在大的空间尺度上绘制栖息地结构的 优势与传统光学遥感利用光谱信息识别地物的优 势相结合, 能进一步提高制图精度, 为物种生境和 生物多样性监测和评估提供更多变量信息(Vierling et al, 2008, 2013; Zellweger et al, 2013)。

例如Nijland等(2015)将光学遥感和机载激光雷 达等多源遥感数据融合, 通过机载激光雷达获取的 森林冠层结构数据与光学遥感获取的树种组成数 据结合, 采用决策树分类方法, 构建了加拿大西部 落基山脉地区灰熊(Rsus americanus altifrontalis)栖 息地立体化的生境分类图, 为灰熊栖息地的生境划 分管理提供更了多元化的信息。Guo等(2017)通过机 载激光雷达获得加拿大阿尔伯塔省不同自然分区 自然分类区植被三维结构信息，通过聚类分析对整 个研究区的植被结构进行制图, 得到其相关的空间 分布, 解决了生物多样性监测中生境结构的基本问 题, 这些可以作为该研究区的本底数据, 与物种和 土地覆盖数据一起用于森林资源规划、物种分布和 动物运动建模, 以及优化关键栖息地结构的保护工 作。Martinuzzi等(2009)通过LiDAR获取的数据与森 林样方调查和随机森林算法三者结合, 得到4种鸟 类重要生境相关的林下层灌木盖度和枯立木胸径 空间异质性分布并制图。Rachlow等(2014)通过地基 雷达获取的数据, 从植被结构的功能属性(被捕食 风险一一视野能见度)出发进行制图, 从捕食者的 视线或者潜在捕食者观察猎物位置的视线, 在景观 尺度上估计捕食者隐藏的有利位置。该研究表明地 基雷达为探索栖息地的变化对猎物和捕食者的潜 在影响提供了全面的数据。Coops等(2016)通过 LiDAR 获取的点云数据, 提取并反演了有关三维生 境的水平和垂直方向的量化指标，即树高、郁闭度、 垂直结构复杂度, 并对这些指标进行空间制图, 用 于物种生境质量和鸟类丰富度的监测评估。

上述研究说明在野生动物栖息地评估方面, 这 些新的基于LiDAR技术的制图有效地改进了以往 依赖于传统光学遥感生境制图的模式, 为栖息地的 监测和评估提供了更多元化和立体化的生境信息。

\section{3 生物多样性评估与物种分布模型预测}

生境结构的异质性作为生物多样性驱动的重 要因素和物种生境质量预测的主要对象, 在森林生 态系统中体现得尤为明显(Zellweger et al, 2016)。以 往由于森林植被三维结构测量困难, 使得生物多样 性和物种分布模拟预测存在很大局限性，而激光雷 达遥感能够在较大的空间尺度上表征动物物种多 样性的关键驱动因素一一栖息地结构, 因此适用于 生物多样性评估和监测(Simonson et al, 2014)。

目前许多研究将LiDAR的三维制图技术用于 生物多样性的监测与物种分布模拟研究(Bergen et al, 2009)。许多研究通过LiDAR获取的点云数据提 取了一系列的植被三维结构信息，获得了与植被结 构密切相关的生物多样性热点区域或高精度的生 境质量预测和模拟(Müller \& Brandl, 2009; Lindberg et al, 2015; Zellweger et al, 2016; Listopad et al, 2018)。Clawges等(2008)通过LiDAR数据计算得到 的植被高度多样性与鸟类物种分布多样性指数呈 显著正相关，从LiDAR数据中提取得到的植被体积 指数与鸟类分布密度也具有明显相关性, 并推测出 潜在的鸟类聚集分布区。Goetz等(2010)利用激光雷 达波形数据定量测量了美国东北部阔叶林植被结 构异质性，确定了冠层垂直分布和复杂度可作为岛 类多年生境利用的重要预测因子, 并且通过LiDAR 获取的系统冠层结构变量与其他生境变量互为补 充，大大提高了鸟类生境利用模型预测的精度。 Zellweger等(2016)从机载激光雷达获取植被结构数 据, 提高了对蝴蝶和鸟类物种丰富度的预测精度, 进一步加强了我们对森林景观中生态位驱动的物 种集群过程的理解。周中一等(2018)以美国加州内 华达山脉南部地区的食鱼貂(Martes pennanti)为例, 探索LiDAR技术在物种分布模拟中的有效性，该研 究表明，当考虑LiDAR变量后，食鱼貂的空间分布 预测精度得到明显提高。这说明LiDAR在物种分布 模拟方面具有很大的潜力。

如何从LiDAR获取的三维植被结构数据中进 一步揭示生境结构异质性与生物多样性或物种分 布的关系, 是未来生物多样性研究的重点。

\section{展望}

由于LiDAR技术提取和反演的森林参数水平 和精度的提高, 越来越多的国外学者将该技术用于 
动物生境质量和动物多样性的研究, 相比传统方法, 提高了动物生境质量的监测水平, 同时也加深了对 生态位驱动的物种生境选择和集群过程的理解, 而 国内利用该技术在动物生态学领域的研究较少, 尚 未见文章发表。

从当前国外的研究现状看, LiDAR技术的应用 主要集中在对已知或潜在的生态关系的研究上, 例 如, 生境结构的异质性与动物多样性的关系, 冠层 结构与鸟类或灵长类动物生境选择的关系等。缺少 对森林生态系统垂直结构, 尤其是冠层以下植被三 维结构异质性的量化和评估, 导致对林下环境的野 生动物生境选择及其与环境的相互作用机理研究 相对缺乏。

因此, 对于我国学者而言, 应当结合国外研究 经验和我国物种保护现状的实际需求, 充分运用 LiDAR技术在精细量化森林生态系统复杂性和异 质性方面的优势, 加强对国家级自然保护区和濒危 物种生境的监测和评估。除了运用LiDAR提取的森 林参数(表3)用于量化和评估动物的生境质量外, 还 需要进一步提取和反演除乔木以外的林下灌草层 垂直结构和生态系统功能参数, 以及具有动物生态 功能和意义的参数(Coops et al, 2016), 从更深的机 理层面探讨由生态位驱动的动物与环境的关系。例 如, 通过LiDAR反演与食草动物食物资源密切相关 的林下灌草层生物量分布信息, 获得食物资源要素 对食草动物分布的影响; 此外还可以建立与动物运 动模式、捕食地点选择、躲避捕食者或睡眠地选择 直接相关的林下植被结构复杂度信息, 进一步加深 对野生动物与环境之间相互关系的认识。

由于LiDAR技术在提取和反演不同尺度的森 林参数方面具有很大的发展潜力, 目前从LiDAR提 取的众多森林参数中有些对动物生境质量和生物 多样性的量化评估具有重要意义, 然而还存在一些 潜在未提取和反演的森林三维结构参数。因此如何 从众多的参数中选出在不同尺度森林生态系统中, 生境质量和生物多样性监测和评估的统一量化指 标及其标准阈值，是下一步研究的关键。将LiDAR 提取的参数进行制图并与动物空间分布数据结合, 通过统计方法分析与生境质量和生物多样性恢复 相关的森林参数条件阈值, 可为不同尺度下生境质 量和生物多样性监测和评估提供量化标准。

综上, LiDAR技术提取和反演的森林参数水平
和精度的提高, 能够较为全面地提供动物三维生境 结构信息, 已经在物种生境选择、生物多样性监测 和生境质量评估以及栖息地生境立体制图方面发 挥了重大的作用。而目前我国在动物生态方面应用 LiDAR技术的研究较少, 未来应加强LiDAR技术对 林下层结构参数的提取和反演, 以及林下层野生动 物生境质量和生物多样性的量化评估，同时建立不 同尺度下的统一量化参数和阈值, 在生境质量评估 和生物多样性监测方面构建一个映射清晰的网络。

\section{参考文献}

Ameztegui A, Coll L (2015) Herbivory and seedling establishment in Pyrenean forests: Influence of micro- and mesohabitat factors on browsing pressure. Forest Ecology and Management, 342, 103-111.

Asner GP, Levick SR (2012) Landscape-scale effects of herbivores on treefall in African savannas. Ecology Letters, 15, 1211-1217.

Bergen KM, Goetz SJ, Dubayah RO, Henebry GM, Hunsaker CT, Imhoff ML, Nelson RF, Parker GG, Radeloff VC (2009) Remote sensing of vegetation 3-D structure for biodiversity and habitat: Review and implications for lidar and radar spaceborne missions. Journal of Geophysical Research: Biogeosciences, 114, G00E06.

Bergl RA, Warren Y, Nicholas A, Dunn A, Imong I, Sunderland-Groves JL, Oates JF (2012) Remote sensing analysis reveals habitat, dispersal corridors and expanded distribution for the Critically Endangered Cross River Gorilla Gorilla gorilla diehli. Oryx, 46, 278-289.

Blakey RV, Law BS, Kingsford RT, Stoklosa J (2017) Terrestrial laser scanning reveals below-canopy bat trait relationships with forest structure. Remote Sensing of Environment, 198, 40-51.

Bradbury RB, Hill RA, Mason DC, Hinsley SA, Wilson JD, Balzter H, Anderson GQA, Whittingham MJ, Davenport IJ, Bellamy PE (2005) Modelling relationships between birds and vegetation structure using airborne LiDAR data: A review with case studies from agricultural and woodland environments. Ibis, 147, 443-452.

Burkepile DE, Burns CE, Tambling CJ, Amendola E, Buis GM, Govender N, Nelson V, Thompson DI, Zinn AD, Smith MD (2013) Habitat selection by large herbivores in a southern African savanna: The relative roles of bottom-up and top-down forces. Ecosphere, 4, art139.

Burton AC, Neilson E, Moreira D, Ladle A, Steenweg R, Fisher JT, Bayne E, Boutin S (2015) REVIEW: Wildlife camera trapping: A review and recommendations for linking surveys to ecological processes. Journal of Applied Ecology, 52, 675-685.

Carlson A (2000) The effect of habitat loss on a deciduous for- 
est specialist species: The white-backed woodpecker (Dendrocopos leucotos). Forest Ecology and Management, 131, 215-221.

Chen J, Mroz GD, Franklin JF, Brosofske KD, Saunders SC, Crow TR, Naiman RJ, Brookshire BL (1999) Microclimate in forest ecosystem and landscape ecology: Variations in local climate can be used to monitor and compare the effects of different management regimes. BioScience, 49, 288-297.

Chen Q, Baldocchi D, P Gong, M Kelly (2006) Isolating individual trees in a savanna woodland using small footprint LiDAR data. Photogrammetric Engineering and Remote Sensing, 72, 923-932.

Chu YMR, Sha JCM, Kawazoe T, Dong X (2018) Sleeping site and tree selection by Sichuan snub-nosed monkeys (Rhinopithecus roxellana) in Baihe Nature Reserve, Sichuan, China. American Journal of Primatology, 80, e22936.

Clark ML, Roberts DA, Ewel JJ, Clark DB (2011) Estimation of tropical rain forest aboveground biomass with smallfootprint lidar and hyperspectral sensors. Remote Sensing of Environment, 115, 2931-2942.

Clawges R, Vierling K, Vierling L, Rowell E (2008) The use of airborne lidar to assess avian species diversity, density, and occurrence in a pine/aspen forest. Remote Sensing of Environment, 112, 2064-2073.

Coops NC, Hilker T, Wulder MA, St-Onge B, Newnham G, Siggins A, Trofymow JA (2007) Estimating canopy structure of Douglas-fir forest stands from discrete-return LiDAR. Trees, 21, 295-310.

Coops NC, Tompaski P, Nijland W, Rickbeil GJM, Nielsen SE, Bater CW, Stadt JJ (2016) A forest structure habitat index based on airborne laser scanning data. Ecological Indicators, 67, 346-357.

Cui LW, Quan RC, Xiao W (2006) Sleeping sites of black-and-white snub-nosed monkeys (Rhinopithecus bieti) at Baima Snow Mountain, China. Journal of Zoology, 270, 192-198.

Davies AB, Ancrenaz M, Oram F, Asner GP (2017) Canopy structure drives orangutan habitat selection in disturbed Bornean forests. Proceedings of the National Academy of Sciences, USA, 114, 8307-8312.

Davies AB, Asner GP (2014) Advances in animal ecology from 3D-LiDAR ecosystem mapping. Trends in Ecology \& Evolution, 29, 681-691.

Davies AB, Oram F, Ancrenaz M, Asner GP (2019) Combining behavioural and LiDAR data to reveal relationships between canopy structure and orangutan nest site selection in disturbed forests. Biological Conservation, 232, 97-107.

Davies AB, Tambling CJ, Kerley GIH, Asner GP (2016) Effects of vegetation structure on the location of lion kill sites in African Thicket. PLoS ONE, 11, e0149098.

Ewald M, Dupke C, Heurich M, Müller J, Reineking B (2014) LiDAR remote sensing of forest structure and GPS telemetry data provide insights on winter habitat selection of
European Roe Deer. Forests, 5, 1374-1390

Flaspohler DJ, Giardina CP, Asner GP, Hart P, Price J, Lyons CK, Castaneda X (2010) Long-term effects of fragmentation and fragment properties on bird species richness in Hawaiian forests. Biological Conservation, 143, 280-288.

Garabedian JE, McGaughey RJ, Reutebuch SE, Parresol BR, Kilgo JC, Moorman CE, Peterson MN (2014) Quantitative analysis of woodpecker habitat using high-resolution airborne LiDAR estimates of forest structure and composition. Remote Sensing of Environment, 145, 68-80.

Garabedian JE, Moorman CE, Nils Peterson M, Kilgo JC (2017) Use of LiDAR to define habitat thresholds for forest bird conservation. Forest Ecology and Management, 399, 24-36.

Garber PA (1987) Foraging strategies among living primates. Annual Review of Anthropology, 16, 339-364.

Goetz SJ, Steinberg D, Betts MG, Holmes RT, Doran PJ, Dubayah R, Hofton M (2010) Lidar remote sensing variables predict breeding habitat of a Neotropical migrant bird. Ecology, 91, 1569-1576.

Guo QH, Su YJ, Hu TY, Liu J (2018) LiDAR Principles, Processing and Application in Forest Ecology. Higher Education Press, Beijing. (in Chinese) [郭庆华, 苏艳军, 胡 天宇, 刘瑾 (2018) 激光雷达森林生态应用一一理论、方 法及实例. 高等教育出版社, 北京.]

Guo X, Coops NC, Tompalski P, Nielsen SE, Bater CW, John SJ (2017) Regional mapping of vegetation structure for biodiversity monitoring using airborne lidar data. Ecological Informatics, 38, 50-61.

Hyde P, Dubayah R, Peterson B, Blair JB, Hofton M, Hunsaker C, Knox R, Walker W (2005) Mapping forest structure for wildlife habitat analysis using waveform lidar: Validation of montane ecosystems. Remote Sensing of Environment, 96, 427-437.

Hyde P, Dubayah R, Walker W, Blair JB, Hofton M, Hunsaker C (2006) Mapping forest structure for wildlife habitat analysis using multi-sensor (LiDAR, SAR/InSAR, ETM+, Quickbird) synergy. Remote Sensing of Environment, 102, 63-73.

Hyyppa J, Kelle O, Lehikoinen M, Inkinen M (2001) A segmentation-based method to retrieve stem volume estimates from 3-D tree height models produced by laser scanners. IEEE Transactions on Geoscience and Remote Sensing, 39, 969-975.

Jaskierniak D, Lane PNJ, Robinson A, Lucieer A (2011) Extracting LiDAR indices to characterise multilayered forest structure using mixture distribution functions. Remote Sensing of Environment, 115, 573-585.

Koch B, Heyder U, Weinacker H (2006) Detection of individual tree crowns in airborne LiDAR data. Photogrammetric Engineering and Remote Sensing, 72, 357-363.

Kwak D, Lee W-K, Cho H-K (2012) Estimation of LAI using LIDAR remote sensing in forest. ISPRS Workshop on Laser 
Scanning, 61, 248-252.

Li W, Q Guo, M Jakubowski, M Kelly (2012) A new method for segmenting individual trees from the Lidar point cloud. Photogrammetric Engineering and Remote Sensing, 78, 75-84.

Li ZY, Liu QW, Pang Y (2016) Review on forest parameters inversion using LiDAR. Journal of Remote Sensing, 20, 1138-1150. (in Chinese with English abstract) [李增元, 刘 清旺, 庞勇 (2016) 激光雷达森林参数反演研究进展. 遥 感学报, 20, 1138-1150.]

Lindberg E, Roberge J-M, Johansson T, Hjältén J (2015) Can airborne laser scanning (ALS) and forest estimates derived from satellite images be used to predict abundance and species richness of birds and beetles in boreal forest? Remote Sensing, 7, 4233-4252.

Listopad CMCS, Köbel M, Príncipe A, Gonçalves P, Branquinho C (2018) The effect of grazing exclusion over time on structure, biodiversity, and regeneration of high nature value farmland ecosystems in Europe. Science of the Total Environment, 610/611, 926-936.

Loarie SR, Tambling CJ, Asner GP (2013) Lion hunting behaviour and vegetation structure in an African savanna. Animal Behaviour, 85, 899-906.

Lone K, Loe LE, Gobakken T, Linnell JDC, Odden J, Remmen J, Mysterud A (2014) Living and dying in a multi-predator landscape of fear: Roe deer are squeezed by contrasting pattern of predation risk imposed by lynx and humans. Oikos, 123, 641-651.

Ma LQ, Li AN (2011) Review of application of LiDAR to estimation of forest vertical structure parameter. World Forestry Research, 24(1), 41-45. (in Chinese with English abstract) [马利群, 李爱农 (2011) 激光雷达在森林垂直 结构参数估算中的应用. 世界林业研究, 24(1), 41-45.]

Martinuzzi S, Vierling LA, Gould WA, Falkowski MJ, Evans JS, Hudak AT, Vierling KT (2009) Mapping snags and understory shrubs for a LiDAR-based assessment of wildlife habitat suitability. Remote Sensing of Environment, 113, 2533-2546.

McDermid GJ, Hall RJ, Sanchez-Azofeifa GA, Franklin SE, Stenhouse GB, Kobliuk T, LeDrew EF (2009) Remote sensing and forest inventory for wildlife habitat assessment. Forest Ecology and Management, 257, 2262-2269.

McGill BJ, Enquist BJ, Weiher E, Westoby M (2006) Rebuilding community ecology from functional traits. Trends in Ecology \& Evolution, 21, 178-185.

McLean KA, Trainor AM, Asner GP, Crofoot MC, Hopkins ME, Campbell CJ, Martin RE, Knapp DE, Jansen PA (2016) Movement patterns of three arboreal primates in a Neotropical moist forest explained by LiDAR-estimated canopy structure. Landscape Ecology, 31, 1849-1862.

Melin M, Matala J, Mehtätalo L, Pusenius J, Packalen P (2016b) Ecological dimensions of airborne laser scanningAnalyzing the role of forest structure in moose habitat use within a year. Remote Sensing of Environment, 173, 238-247.

Melin M, Mehtätalo L, Miettinen J, Tossavainen S, Packalen P (2016a) Forest structure as a determinant of grouse brood occurrence-An analysis linking LiDAR data with presence/absence field data. Forest Ecology and Management, 380, 202-211.

Morsdorf F, Meier E, Koetz B, Itten K, Dobbertin M, Allgöwer B (2004) LIDAR-based geometric reconstruction of boreal type forest stands at single tree level for forest and wildland fire management. Remote Sensing of Environment, 92, 353-362.

Müller J, Bae S, Röder J, Chao A, Didham RK (2014) Airborne LiDAR reveals context dependence in the effects of canopy architecture on arthropod diversity. Forest Ecology and Management, 312, 129-137.

Müller J, Brandl R (2009) Assessing biodiversity by remote sensing in mountainous terrain: The potential of LiDAR to predict forest beetle assemblages. Journal of Applied Ecology, 46, 897-905.

Nijland W, Coops NC, Nielsen SE, Stenhouse G (2015) Integrating optical satellite data and airborne laser scanning in habitat classification for wildlife management. International Journal of Applied Earth Observation and Geoinformation, 38, 242-250.

Olsoy PJ, Shipley LA, Rachlow JL, Forbey JS, Glenn NF, Burgess MA, Thornton DH (2018) Unmanned aerial systems measure structural habitat features for wildlife across multiple scales. Methods in Ecology and Evolution, 9, 594-604.

Palminteri S, Powell GVN, Asner GP, Peres CA (2012) LiDAR measurements of canopy structure predict spatial distribution of a tropical mature forest primate. Remote Sensing of Environment, 127, 98-105.

Pang Y, Zhao F, Li ZY, Zhou SF, Deng G, Liu QW, Chen EX (2008) Forest height inversion using airborne LiDAR technology. Journal of Remote Sensing, 12, 152-158. (in Chinese with English abstract) [庞勇, 赵峰, 李增元, 周淑 芳, 邓广, 刘清旺, 陈尔学 (2008) 机载激光雷达平均树 高提取研究. 遥感学报, 12, 152-158.]

Psyllakis JM, Gillingham MP (2009) Using forest structure and composition to predict the occurrence of vertebrate species in Douglas-Fir forests of British Columbia. Biological Conservation, 142, 1427-1441.

Rachlow JL, Forbey JS, Nobler JD, Shipley LA, Glenn NF, Olsoy PJ (2014) Fearscapes: Mapping functional properties of cover for prey with terrestrial LiDAR. BioScience, 65, 74-80.

Reitberger J, C Schnörr, P Krzystek, U Stilla (2009) 3D segmentation of single trees exploiting full waveform LIDAR data. ISPRS Journal of Photogrammetry and Remote Sensing, 64, 561-574.

Ren B, Zhang S, Wang L, Liang B, Li B (2001) Vertical 
distribution of different age-sex classes in a foraging group of Sichuan Golden Monkeys (Rhinopithecus roxellana). Folia Primatologica, 72, 96-99.

Ripple WJ, Chapron G, López-Bao JV, Durant SM, Macdonald DW, Lindsey PA, Bennett EL, Beschta RL, Bruskotter JT, Campos-Arceiz A, Corlett RT, Darimont CT, Dickman AJ, Dirzo R, Dublin HT, Estes JA, Everatt KT, Galetti M, Goswami VR, Hayward MW, Hedges S, Hoffmann M, Hunter LTB, Kerley GIH, Letnic M, Levi T, Maisels F, Morrison JC, Nelson MP, Newsome TM, Painter L, Pringle RM, Sandom CJ, Terborgh J, Treves A, Van Valkenburgh B, Vucetich JA, Wirsing AJ, Wallach AD, Wolf C, Woodroffe R, Young H, Zhang L (2016) Saving the world's terrestrial megafauna. BioScience, 66, 807-812.

Saracco JF, Collazo JA, Groom MJ (2004) How do frugivores track resources? Insights from spatial analyses of bird foraging in a tropical forest. Oecologia, 139, 235-245.

Serrouya R, D’Eon RG (2008) The influence of forest cover on mule deer habitat selection, diet, and nutrition during winter in a deep-snow ecosystem. Forest Ecology and Management, 256, 452-461.

Simonson WD, Allen HD, Coomes DA (2014) Applications of airborne lidar for the assessment of animal species diversity. Methods in Ecology and Evolution, 5, 719-729.

Takahata C, Nielsen SE, Takii A, Izumiyama S (2014) Habitat selection of a large carnivore along human-wildlife boundaries in a highly modified landscape. PLoS ONE, 9, e86181.

Tao S, Wu F, Guo Q, Wang Y, Li W, Xue B, Hu X, Li P, Tian D, Li C, Yao H, Li Y, Xu G, Fang J (2015) Segmenting tree crowns from terrestrial and mobile LiDAR data by exploring ecological theories. ISPRS Journal of Photogrammetry and Remote Sensing, 110, 66-76.

Thomas V, Treitz P, McCaughey H, Morrison I (2006) Mapping stand-level forest biophysical variables for a mixedwood boreal forest using LiDAR: An examination of scanning density. Canadian Journal of Forest Research, 36, 34-47.

Vermeulen C, Lejeune P, Lisein J, Sawadogo P, Bouché P (2013) Unmanned aerial survey of elephants. PLoS ONE, 8, e54700.

Vierling KT, Vierling LA, Gould WA, Martinuzzi S, Clawges RM (2008) Lidar: Shedding new light on habitat characterization and modeling. Frontiers in Ecology and the Environment, 6, 90-98.

Vierling LA, Vierling KT, Adam P, Hudak AT (2013) Using satellite and airborne LiDAR to model woodpecker habitat occupancy at the landscape scale. PLoS ONE, 8, e80988.

Wang YY, Wang JW, Zhao LL (2018) Research on 3D digital technology of building based on LiDAR data. Journal of Shandong Jianzhu University, 33, 79-84. (in Chinese with English abstract) [王瑶瑶, 王京卫, 赵莉莉 (2018) 基于 激光雷达的建筑三维数字化技术应用研究. 山东建筑大 学学报, 33, 79-84.]

Xiang ZF, Nie SG, Chang ZF, Wei FW, Li M (2010) Sleeping sites of Rhinopithecus brelichi at Yangaoping, Guizhou. International Journal of Primatology, 31, 59-71.

Xiao W, Feng L, Mou P, Miquelle DG, Hebblewhite M, Goldberg JF, Robinson HS, Zhao X, Zhou B, Wang T, Ge J (2016) Estimating abundance and density of Amur tigers along the Sino-Russian border. Integrative Zoology, 11, 322-332.

Zellweger F, Baltensweiler A, Ginzler C, Roth T, Braunisch V, Bugmann H, Bollmann K (2016) Environmental predictors of species richness in forest landscapes: Abiotic factors versus vegetation structure. Journal of Biogeography, 43, 1080-1090.

Zellweger F, Braunisch V, Baltensweiler A, Bollmann K (2013) Remotely sensed forest structural complexity predicts multi species occurrence at the landscape scale. Forest Ecology and Management, 307, 303-312.

Zhang J, Zhang L, Zeng F, Wang X, Zhao JX, Tian HY, Ren H, Li JF (2011) Development status of airborne 3D imaging lidar systems. Chinese Optics, 4, 213-232. (in Chinese with English abstract) [张健, 张雷, 曾飞, 王旭, 赵嘉金金, 田海 英, 任航, 李俊峰 (2011) 机载激光3D探测成像系统的发 展现状. 中国光学, 4, 213-232.]

Zhao K, García M, Liu S, Guo Q, Chen G, Zhang X, Zhou Y, Meng X (2015) Terrestrial lidar remote sensing of forests: Maximum likelihood estimates of canopy profile, leaf area index, and leaf angle distribution. Agricultural and Forest Meteorology, 209/210, 100-113.

Zhou ZY, Liu R, Shi SN, Su YJ, Li WK, Guo QH (2018) Ecological niche modeling with LiDAR data: A case study of modeling the distribution of fisher in the southern Sierra Nevada Mountains, California. Biodiversity Science, 26, 878-891. (in Chinese with English abstract) [周中一, 刘手, 时书纳, 苏艳军, 李文楷, 郭庆华 (2018) 基于激光雷达 数据的物种分布模拟：以美国加州内华达山脉南部区域 食鱼貂分布模拟为例. 生物多样性, 26, 878-891.]

(责任编委: 郭庆华 责任编辑: 时意专) 